\title{
Formation and dissociation of proteasome storage granules are regulated by cytosolic $\mathrm{pH}$
}

\author{
Lee Zeev Peters, ${ }^{1}$ Rotem Hazan, ${ }^{1}$ Michal Breker, ${ }^{2}$ Maya Schuldiner, ${ }^{2}$ and Shay Ben-Aroya ${ }^{1}$ \\ 'The Nano Center, Faculty of Life Sciences, Bar-llan University, Ramat-Gan 52900, Israel \\ ${ }^{2}$ Department of Molecular Genetics, Weizmann Institute of Science, Rehovot 76100, Israel
}

$\mathrm{T}$ he $26 \mathrm{~S}$ proteasome is the major protein degradation machinery of the cell and is regulated at many levels. One mode of regulation involves accumulation of proteasomes in proteasome storage granules (PSGs) upon glucose depletion. Using a systematic robotic screening approach in yeast, we identify trans-acting proteins that regulate the accumulation of proteasomes in PSGs. Our dataset was enriched for subunits of the vacuolar adenosine triphosphatase (V-ATPase) complex, a proton pump required for vacuole acidification.
We show that the impaired ability of V-ATPase mutants to properly govern intracellular $\mathrm{pH}$ affects the kinetics of PSG formation. We further show that formation of other protein aggregates upon carbon depletion also is triggered in mutants with impaired activity of the plasma membrane proton pump and the V-ATPase complex. We thus identify cytosolic $\mathrm{pH}$ as a specific cellular signal involved both in the glucose sensing that mediates PSG formation and in a more general mechanism for signaling carbon source exhaustion.

\section{Introduction}

The $26 \mathrm{~S}$ proteasome is a $2.5-\mathrm{MD}$ protease that is composed of the $19 \mathrm{~S}$ regulatory particle (RP) and the $20 \mathrm{~S}$ core particle (CP), which possesses the protease activity. In eukaryotes, the major mechanism for targeting proteins to the proteasome is through the process of ubiquitination. Through this pathway the ubiquitinproteasome system exerts its influence by selectively degrading misfolded, damaged, or regulatory proteins (Hershko and Ciechanover, 1998).

Proteolytic function must be tightly regulated in a cellular environment, and therefore, mechanisms exist to turn the proteasome off. This massive complex is highly abundant $(\sim 1 \%$ of the yeast proteome; Russell et al., 1999) and therefore costly to synthesize and assemble. Therefore, degradation can be considered to be the least favored regulation in this case. The alternate option, that is widely used, is to assemble it into proteasome storage granules (PSGs) during cellular stress conditions such as glucose starvation or quiescence (Laporte et al., 2008). In these conditions, nonselective autophagy is activated in yeast cells, and it is thought that the PSGs represent a protective mechanism that shelters the proteasome from autophagic degradation.

L.Z. Peters, R. Hazan, and M. Breker contributed equally to this paper. Correspondence to Shay Ben-Aroya: Shay.Ben-Aroya@biu.ac.il

Abbreviations used in this paper: CCCP, carbonyl cyanide 3-chlorophenylhydrazone; $\mathrm{CP}$, core particle; $\mathrm{DIC}$, differential interference contrast; $\mathrm{pHi}$, intracellular $\mathrm{pH}$; PSG, proteasome storage granule; RP, regulatory particle; SC, synthetic complete; SD, synthetic defined; SGA, synthetic genetic array; SPG, stationaryphase granule; V-ATPase, vacuolar ATPase; wt, wild type.
The utilization of PSGs enables cells to avoid the replenishment of large amounts of active proteasome subunits and the possible delay in reentry into the proliferative state. In fact, many other proteins that are localized diffusely in the cytoplasm of dividing yeast cells relocalize into cytoplasmic foci upon glucose starvation or entry into quiescence. The widespread reorganization of nuclear and cytoplasmic proteins into reversible assemblies suggests that it is a general mechanism for coping with starvation (Narayanaswamy et al., 2009).

Attempts to comprehend which nutrients or chemicals trigger the formation of cytoplasmic granules showed that depletion or addition of glucose is sufficient and that glucose has to be catabolized through the glycolytic pathway to exert this effect (Daignan-Fornier and Sagot, 2011; Laporte et al., 2011). Still, at the present time, it is not clear which metabolite resulting from glucose catabolism is required for glucose induced cytoplasmic granule mobilization.

In this paper, we describe a whole-genome systematic screening approach that allowed us to discover mutants that affect the shuttling of the proteasome into PSGs (Fig. $1 \mathrm{~A}$ ). The functional distribution of the identified mutants reveals enrichment for subunits of the vacuolar ATPase (V-ATPase; Table 1). We show that

(c) 2013 Peters et al. This article is distributed under the terms of an Attribution-NoncommercialShare Alike-No Mirror Sites license for the first six months after the publication date (see http://www.rupress.org/terms). After six months it is available under a Creative Commons License (Attribution-Noncommercial-Share Alike 3.0 Unported license, as described at http://creativecommons.org/licenses/by-nc-sa/3.0//. 
Figure 1. A systematic approach for the identification of trans-acting proteins regulating proteasome sequestering into PSGs. (A) To uncover novel regulators of proteasome localization into PSGs, we integrated Rpn5-GFP into every strain of the yeast deletion collection (1), resulting in a systematic library harboring the tagged proteasome on the background of single gene deletions (2). After high content screening (3), we manually categorized the localization of Rpn5-GFP for all strains tested (4) and recognized hits. (B) Deletion of V-ATPase subunits results in proteasome failure to leave PSGs during logarithmic growth. Representative images of data acquired during the systematic screening approach described in $A$. Although Rpn5-GFP accumulates in the nucleus in the control cells (wt), it is still found in PSGs in cells deleted for V-ATPase subunits (pointed to by a white arrow in vma22 mutant) despite the abundance of glucose. Bar, $5 \mu \mathrm{m}$.
A
1. Introduction of Rpn5-GFP into the deletion collection

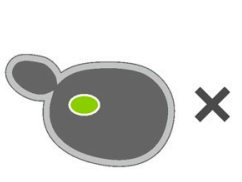

Rpn5-GFP

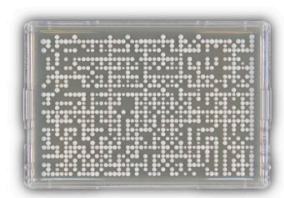

Deletion collection

\section{Automated image acquisition during logarithmic growth}

2. Resulting library harbors Rpn5-GFP at the background of single deletion

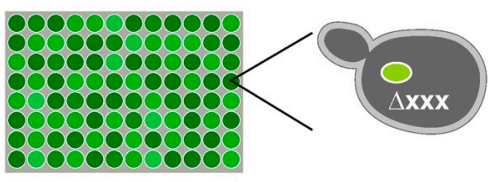

4. Manual image analysis for mutant phenotype

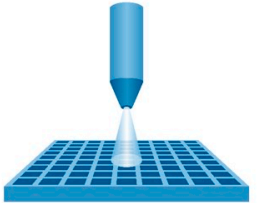

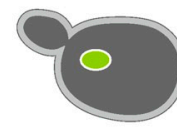

WT
Vs.

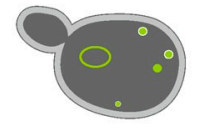

Deletion of interest

B
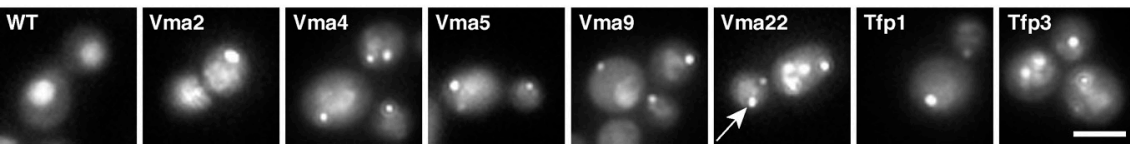

the kinetics of PSG formation in the mutants upon carbon depletion is different from that seen in the wild type (wt), probably because of the impaired ability of the V-ATPase mutants to properly adjust their intracellular $\mathrm{pH}(\mathrm{pHi})$, in response to carbon starvation. Further characterization identified cytosolic $\mathrm{pH}$ as a novel regulator that mediates the formation of PSGs and other protein aggregates that form upon sugar depletion. We therefore propose that cytosolic $\mathrm{pH}$ acts as the cellular signal for glucose sensing and mediates the formation of PSGs in response to carbon depletion.

\section{Results and discussion}

A systematic approach for the identification of trans-acting proteins regulating proteasome sequestering into PSGs

The regulation of proper partitioning of the proteasome into PSGs is essential for maintaining the correct level of the proteasome in the cytosol and nucleus. Therefore, we set out to uncover additional factors that regulate this process. To visually screen for such factors, we first created a yeast strain (based on the yeast S288C background; Brachmann et al., 1998) expressing a fluorescently tagged RP proteasome subunit (Rpn5-GFP). Indeed, as expected, this fusion protein localized correctly to both the cytosol and the nucleus (Fig. 1 B, wt). Using synthetic genetic array (SGA) methodology (Tong and Boone, 2006; Cohen and Schuldiner, 2011), we crossed this strain against the yeast deletion library (Giaever et al., 2002), which consists of $\sim 5,000$ strains, each harboring a deletion in one nonessential gene. This procedure allowed us to create a library of nearly 5,000 haploid cells, each expressing the Rpn5-GFP fusion protein on the background of a mutation in a single gene (Fig. 1 A). The freshly grown colonies were copied from agar plates into liquid cultures in synthetic medium containing glucose (synthetic defined [SD]) for overnight growth. After back dilution into fresh medium for

Table 1. List of genes identified through deletion mutants affecting proteasome sequestering into PSGs

\begin{tabular}{|c|c|c|}
\hline Description & Gene name & ORF name \\
\hline Subunit $d$ of the five-subunit VO integral membrane domain of V-ATPase & VMA6 & YLR447C \\
\hline Peripheral membrane protein that is required for V-ATPase function & VMA22 & YHRO6OW \\
\hline V-ATPase VO domain subunit $c^{\prime}$, involved in proton transport activity; hydrophobic integral membrane protein & TFP3 & YPL234C \\
\hline Subunit $C$ of the eight-subunit $V 1$ peripheral membrane domain of V-ATPase & VMA5 & YKLO80W \\
\hline Subunit B of the eight-subunit V1 peripheral membrane domain of the V-ATPase & VMA2 & YBR 127C \\
\hline $\begin{array}{l}\text { Dubious ORF; ORF overlaps the verified gene VMA4/YOR332W: subunit } \mathrm{E} \text { of the eight-subunit V1 peripheral } \\
\text { membrane domain of the V-ATPase }\end{array}$ & YOR331C & YOR331C \\
\hline Subunit A of the eight-subunit V1 peripheral membrane domain of the V-ATPase & TFP1 & YDL185W \\
\hline Dubious ORF; overlaps verified ORF YCL005W-A IVMA9: V-ATPase subunit e of the V-ATPase V0 subcomplex & YCLOO7C & YCLOOTC \\
\hline $\begin{array}{l}\text { DNA damage-inducible V-SNARE binding protein contains a ubiquitin-associated domain, may act as a } \\
\text { negative regulator of constitutive exocytosis, and may play a role in S-phase checkpoint control }\end{array}$ & DDII & YER I 43W \\
\hline $\begin{array}{l}\text { Component of the GTPase-activating Bfalp-Bub2p complex involved in multiple cell cycle checkpoint } \\
\text { pathways that control exit from mitosis }\end{array}$ & $B F A l$ & YJR053W \\
\hline $\begin{array}{l}\text { Protein that stimulates the activity of serine palmitoyltransferase (Lcb lp and Lcb2p) severalfold; involved in } \\
\text { sphingolipid biosynthesis }\end{array}$ & TSC3 & YBR058C-A \\
\hline
\end{tabular}


$3.5 \mathrm{~h}$, cells were transferred into glass-bottom 384-well microscope plates. We then visualized the cellular localization of Rpn5GFP in each of these mutated strains during logarithmic growth in SD using a high throughput microscopy setup (for details see Materials and methods; Fig. 1 A; Cohen and Schuldiner, 2011). The pictures were then manually visualized to uncover strains in which the normal localization was disturbed.

In the majority of the cases, cells responded as expected to the increase in glucose levels (caused during the back dilution into fresh medium) and relocalized their proteasomes from PSGs back into the cytosol and the nucleus. However, the time of recovery in 11 mutants was significantly delayed, enabling us to identify genes that abrogate the ability of cells to sense the changes in glucose levels and respond properly by releasing proteasomes from PSGs (Table 1). Interestingly, the functional distribution of the identified mutants reveals that our data were highly enriched for genes that govern $\mathrm{pHi}$. These genes include seven subunits of the V-ATPase complex and one subunit required for its assembly (Table 1).

\section{Impaired ability of V-ATPase mutants \\ to control pHi significantly affects \\ the redistribution of the proteasome \\ from PSGs}

V-ATPase, a proton pump required for the acidification of vacuoles, plays a central role in vacuolar and cytosolic $\mathrm{pH}$ homeostasis in all eukaryotic cells. V-ATPases are activated by glucose and function together with Pma1 to pump protons out of the cytosol to help regulate cytosolic $\mathrm{pH}$. Reduction in cytosolic $\mathrm{pH}$ as a result of glucose deprivation is necessary for the inactivation of V-ATPase by their reversible disassembly (Dechant et al., 2010). In V-ATPase mutants, even at optimal extracellular $\mathrm{pH}$, cytosolic $\mathrm{pH}$ was previously shown to be much lower, and cells failed to respond properly when glucose was added (Dechant et al., 2010; Young et al., 2010). Given that it is known that PSGs are regulated by glucose availability, we were intrigued by the idea that V-ATPase function could be the missing link between glucose levels and signaling of PSG formation.

Because the screen was performed at a single time point and measured mostly the dissociation of PSGs, we decided to look at the effect of V-ATPase mutants on the kinetics of PSGs formation. We focused on a representative mutant, $\Delta v m a 2$ and followed the localization of Rpn5-GFP after depletion of glucose relative to control (wt) cells. At $\mathrm{t}=0$, Rpn5-GFP localized to the nucleus both in wt and $\Delta v m a 2$ mutants (Fig. $2 \mathrm{~A}$, $\mathrm{t}=0$ ). Resuspension of wt cells in glucose-free synthetic media (synthetic complete [SC]) led to the expected relocalization of Rpn5-GFP to PSGs in $87 \%$ of the cells within 24 h. Strikingly, in $\Delta v m a 2$ cells, the kinetics of PSGs formation was significantly faster with $44 \%$ of the cells displaying Rpn5-GFP localization to the nuclear periphery after $1 \mathrm{~h}$ and $72 \%$ of the cells displaying PSGs after $<4 \mathrm{~h}$. Furthermore, as expected from the phenotypes observed in our screen, the kinetics of Rpn5-GFP disassembly and relocalization back into the nucleus in response to glucose replenishment was significantly slower in $\Delta v m a 2$ mutants (Fig. 2 A, +Dex; the full kinetics of disassembly and relocalization is shown in Fig. S1 C). Similar kinetics were obtained with cells mutated in another V-ATPase subunit ( $\Delta v$ ma5; Fig. S1 D), with the CP protein Pup2 fused to GFP (Fig. 2 B), and with cells treated with the V-ATPase-specific inhibitor concanamycin A (Fig. S1 E; Krogan et al., 2004; Dechant et al., 2010). We also verified that Pup2 (tagged with mCherry) and Rpn5 (tagged with GFP) colocalize in the PSGs formed in $\Delta v m a 2$ to ensure that this mutation did not create a change in PSG physiology (Fig. S1 F). Collectively, these results suggest that the impaired ability to control pHi in V-ATPase mutants increased the sensitivity of cells to lower levels of the carbon source and induced the simultaneous relocalization of both core and regulatory proteasomal subunits into PSGs (Fig. 2).

Rapid drop of internal pH is sufficient

for formation of PSGs at constant

glucose levels

Our screen identified that mutated subunits of V-ATPase, key regulators of $\mathrm{pHi}$ homeostasis, affect the kinetics of PSGs formation. Previous studies have shown that glucose-starved yeast exhibit a rapid drop in pHi (Martínez-Muñoz and Kane, 2008; Dechant et al., 2010; Young et al., 2010). Because both glucosestarved yeast and mutants that control pHi (Fig. 2) affect the formation of PSGs, we hypothesized that the internal drop in cytosolic $\mathrm{pH}$ might be the physiological signal for PSGs formation. To test this hypothesis, we assayed whether changes in $\mathrm{pHi}$ are sufficient to mediate the formation of PSGs, even when glucose levels are kept high (2\%).

First, we analyzed the localization of Rpn5-GFP at timely intervals, after cells were shifted to dextrose-containing buffers, buffered to normal and low $\mathrm{pH}$ ( $\mathrm{pH} 7.5$ and 4.0, respectively). The buffers also contained the proton ionophore carbonyl cyanide 3-chlorophenylhydrazone (CCCP), which affects the plasma membrane proton gradient and therefore prevents cells from controlling their internal $\mathrm{pH}$ (Orij et al., 2009). The results clearly show that after $120 \mathrm{~min}$, under normal pH (7.5), the GFP signal was mainly localized to the nucleus ( $\sim 63 \%$ of the cells) and, in $\sim 30 \%$ of the cells, to the nuclear periphery (Fig. 3, A [top] and B). In contrast, at a lower $\mathrm{pH}$, the proteasome formed PSGs in 55\% of the cells (Fig. 3 A, bottom, white arrow) and relocalized to the nuclear periphery in the others. When the cells were shifted back to physiological $\mathrm{pH}$ and allowed to recover in the presence of dextrose + CCCP, the GFP signal in most cells relocated back into the nucleus within $30 \mathrm{~min}$ (Fig. $3 \mathrm{~A}$, right). Furthermore, the PSGs formed after $24 \mathrm{~h}$ in minimal medium (Fig. 3 C, left) relocalized back into nucleus (in $95 \%$ of the cells) when cells were resuspended in a medium adjusted to $\mathrm{pH} 7.5$, supplemented with CCCP and glucose (Fig. $3 \mathrm{C}$, middle). In contrast, relocalization was impaired when cells were resuspended in a similar medium that was buffered to a low $\mathrm{pH}$ (4.0; Fig. 3 C, right).

Next, we decided to test the effect of physiological changes in pHi by analyzing the localization of Rpn5-GFP on cells expressing reduced levels of Pma1. It has been previously shown that in wt cells, in response to glucose starvation, Pma1 is repressed, resulting in a rapid drop in pHi (Fig. $4 \mathrm{~A}$, left and middle; Young et al., 2010). pmal-007 is a hypomorphic allele of PMA1 that was previously shown to reduce the activity 
A

Time without carbon source $(\mathrm{h})$
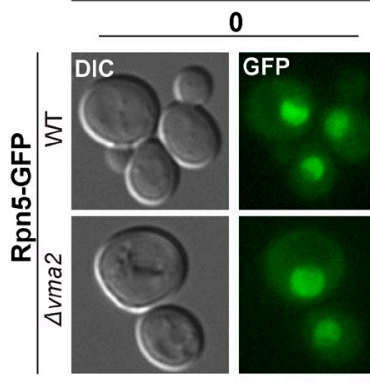
1
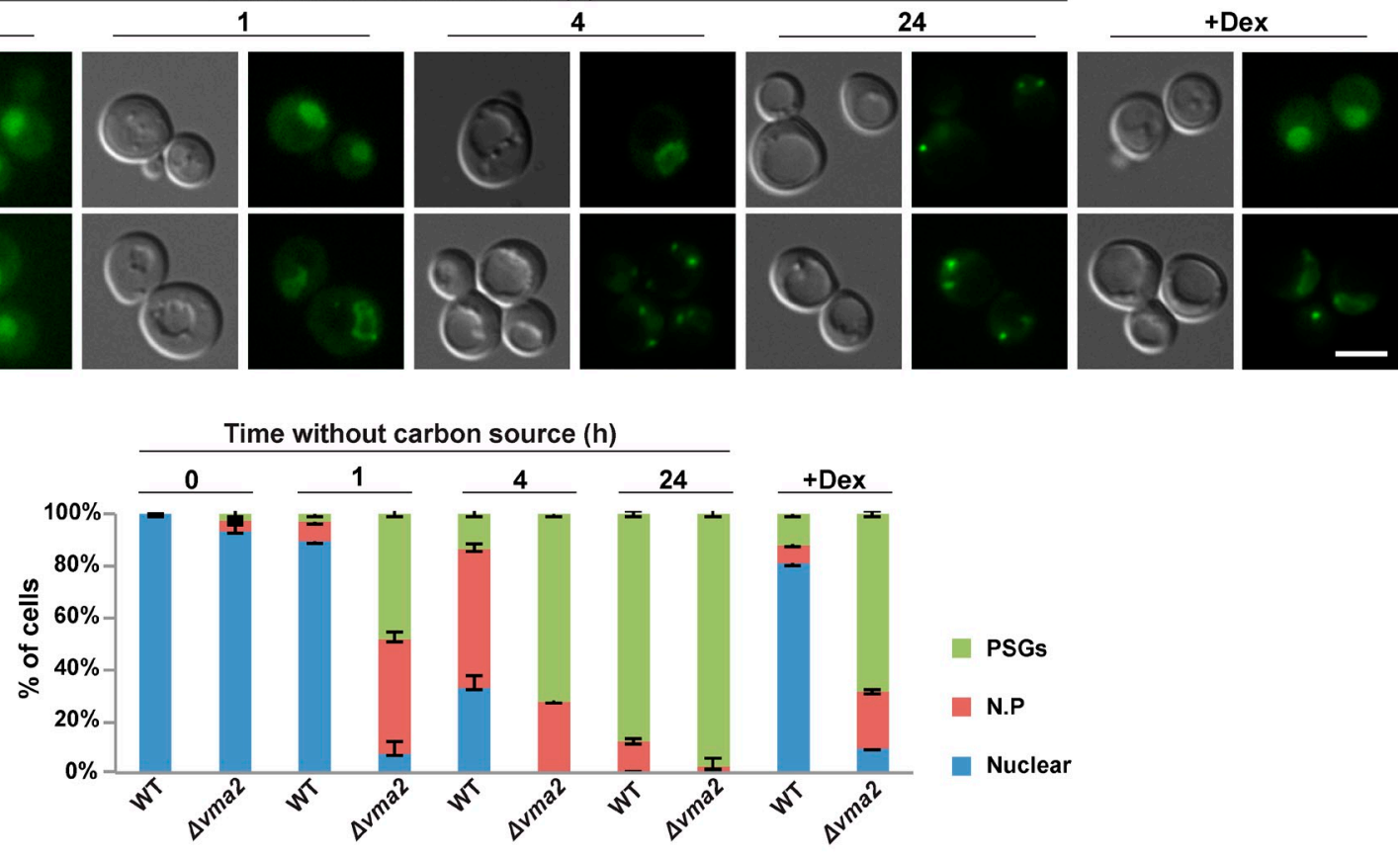

B
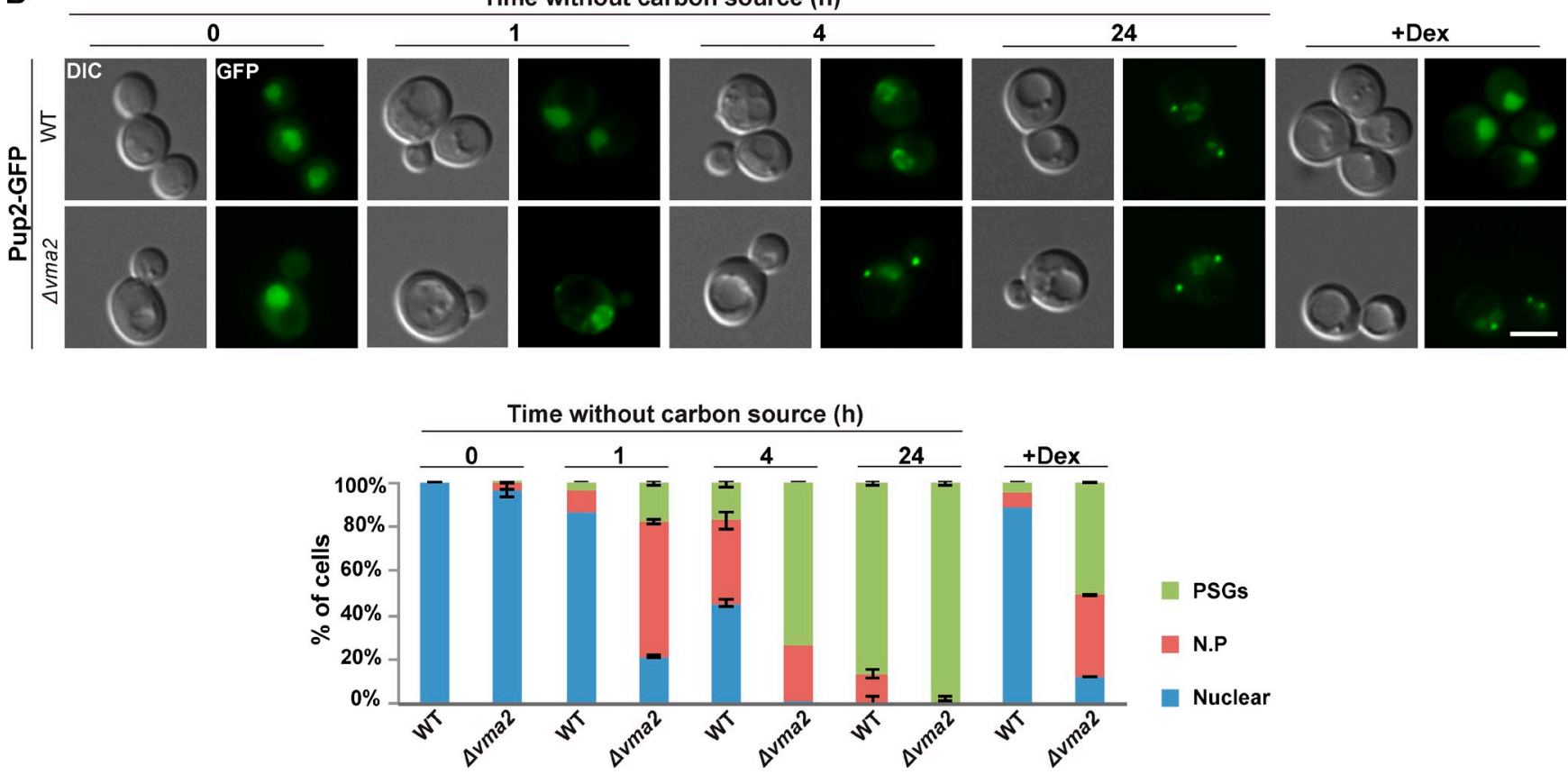

Figure 2. The kinetics of PSG formation is affected in cells deleted in V-ATPase subunit VMA2 ( $\mathbf{v} v a 2)$. (A and B) Logarithmic wt and $\Delta v$ vma2 cells expressing the lid subunit Rpn5-GFP (A) and the core subunit Pup2-GFP (B) were washed and resuspended in glucose-free medium ( $t=0)$ for $24 \mathrm{~h}$ and then transferred back into a glucose-rich medium (+dextrose [+DEX]). Cells were visualized by differential interference contrast (DIC) and GFP at the indicated time points. The localization of Rpn5-GFP (A) and Pup2-GFP (B) was scored as nuclear, nuclear periphery (N.P), or PSGs. Unless otherwise stated, the representative images in all figures show the dominant localization pattern for each of the indicated time points. For each time point, a minimum of 200 cells was counted $(n>200)$; error bars show the standard deviation between two independent experiments. Bars, 5 rm. Images represent high resolution $(63 x)$ images of a single plane chosen from $z$ series images extending above and below the entire cell.

of the Pma1 protein by 50\% (Porat et al., 2005; Young et al., 2010). This results in a decreased capacity to pump protons out of the cell and a concomitant reduction in cytosolic pHi, similar to that which occurs when CCCP is added externally (Fig. 4 A, right). The results clearly show that although both strains were grown in glucose-rich medium for $30 \mathrm{~h}$, the rate of PSG formation was significantly higher in pmal-007 mutants, with $31 \%$ of the cells forming PSGs in the mutants, $(n=$ $210)$ versus only $8 \%$ in the control $(n=204$; Fig. 4 , B and C). Under these conditions, the proteasome CP subunit Pre6-mCherry was similarly affected and clearly colocalized with the Rpn5GFP (Fig. S2 A). To further support these results, we transferred 
A
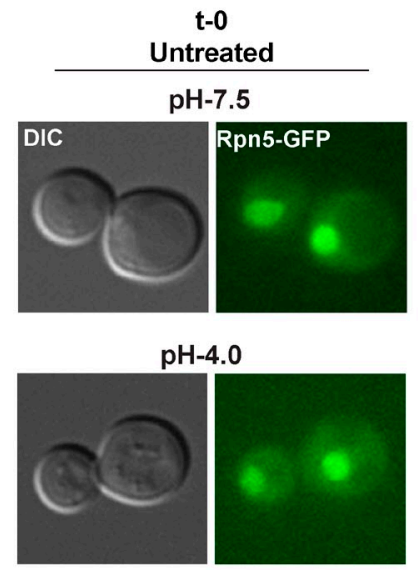
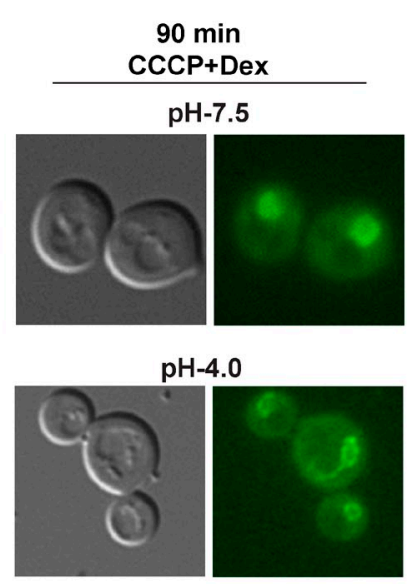
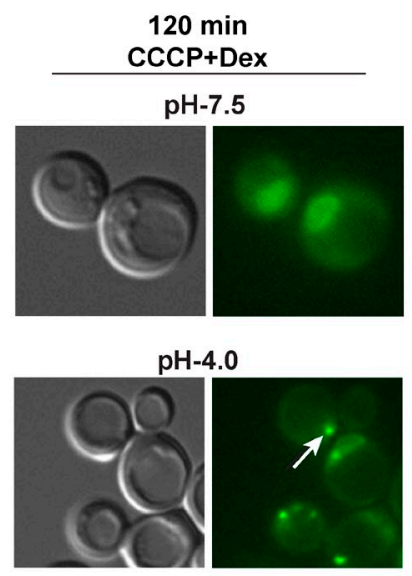

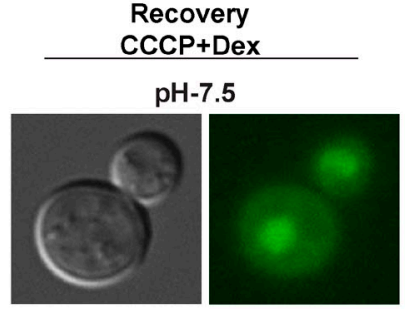

$\mathrm{pH}-7.5$

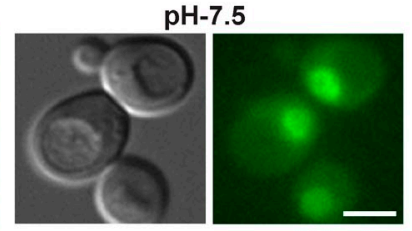

B

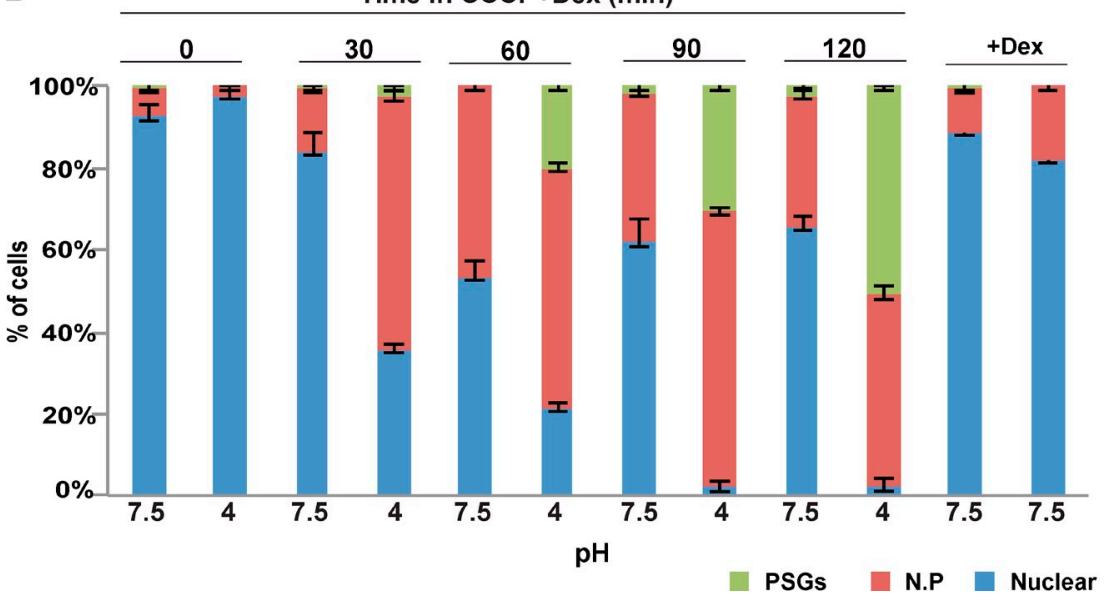

C
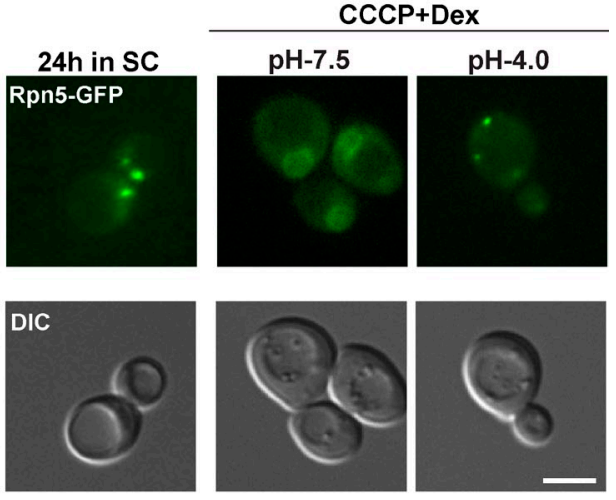

Figure 3. Failure to control internal pH affects PSG formation. (A) Logarithmically growing culture (untreated), expressing the lid subunit Rpn5-GFP, was washed and resuspended in glucose (dextrose [Dex])-containing buffers, buffered to physiological pHi, $\mathrm{pH} 7.5$ (control), and to pH 4.0 , supplemented with CCCP. Both samples were then washed and allowed to recover for $30 \mathrm{~min}$ in $\mathrm{pH} 7.5+\mathrm{CCCP}$ (Recovery). Cells were visualized by DIC and GFP at the indicated time points. The localization of Rpn5-GFP was scored as described in Fig. 2. PSGs are pointed out by a white arrow. (B) Representative images in all figures show the dominant localization pattern for each of the indicated time points. Error bars show the standard deviation between two independent experiments. (C) Similar experimental setup as was described in A, this time, PSG relocalization in cells grown in a low glucose media for $24 \mathrm{~h}$ ( $24 \mathrm{~h}$ in SC) was examined after the resuspension in the indicated medium (CCCP + dextrose). N.P, nuclear periphery. Bars, $5 \mu \mathrm{m}$.

the same strains to a medium that lacks any carbon source. The lack of carbon should result in a faster reduction in $\mathrm{pHi}$ in control cells, and to a greater extent in pmal-007 mutants, when compared with SD. As expected, under such conditions, the kinetics of PSGs formation were dramatically faster and were detected after $4 \mathrm{~h}$, with $62 \%$ of the cells exhibiting PSGs in the mutants, $(n=200)$ versus $18 \%$ in the control $(n=200$; Fig. S2 B). Furthermore, we have repeated the experiment described in Fig. 4 A, this time in the presence of $100 \mu \mathrm{M}$ ebselen, both in wt cells and pmal-007 mutants. A previous study has shown that the drug ebselen inhibits Pmal in vitro and causes an immediate drop in $\mathrm{pHi}$ of both wt and pmal-007 cells to $\sim 6.3$. Whereas wt cells stabilized at $\mathrm{pHi} \sim 6.4$, pHi of pmal007 cells continued to decrease (Chan et al., 2007). As expected from the immediate drop in internal $\mathrm{pHi}$, we have observed that the addition of ebselen resulted in faster kinetics of nuclear periphery and PSG formation in pmal-007 cells, indicating that the mutant is more sensitive, likely as a result of reduced gene dosage (Fig. S2 C).
In total, these results strongly suggest that the rapid drop in $\mathrm{pHi}$ and the acidification of the cytosol (either by external treatment with CCCP or genetically by reducing Pma1 levels) are sufficient to trigger PSG formation. Overall, our findings demonstrate for the first time that the change in cytosolic $\mathrm{pH}$ in response to glucose levels serves as a messenger and a major cellular signal to mediate the activation of PSGs reorganization. $\mathrm{pH}$ homeostasis is critical for the survival of yeast cells, as it is for all eukaryotic cells. Indeed, as expected, previous studies have shown a link between cytosolic $\mathrm{pH}$ and various cellular activities. For example, Young et al., 2010 have demonstrated a link between nutrient availability, $\mathrm{pH}$ biosensing, and membrane biogenesis. A very recent study identified that an early age increase in vacuolar $\mathrm{pH}$ limits mitochondrial function and life span in yeast (Hughes and Gottschling, 2012). Other work has shown that the increase in cytosolic $\mathrm{pH}$ as a result of addition of glucose in starved cells promotes V-ATPase assembly and that this activation positively regulates the cAMP-dependent PKA pathway in yeast (Dechant et al., 2010). Similarly, our study 
Figure 4. Impaired capacity to pump protons out of cells affects the kinetics of PSG formation. (A) Schematic illustration of PMA 1 regulation. (B) pma 1-007 and wt cells were grown in SD for $30 \mathrm{~h}$. Cells were visualized by DIC and GFP at the indicated time points. The representative images shown at $\mathrm{t}=30 \mathrm{~h}$ show the PSG localization pattern that was significantly enriched in pmal-007 mutants, comparing with the nuclear localization pattern that still appeared in wt cells. Bar, 5 um. (C) Cells were visualized by DIC and GFP at the indicated time points. The localization of Rpn5-GFP was scored as described in Fig. 2. Error bars show the standard deviation between two independent experiments. N.P, nuclear periphery.
A

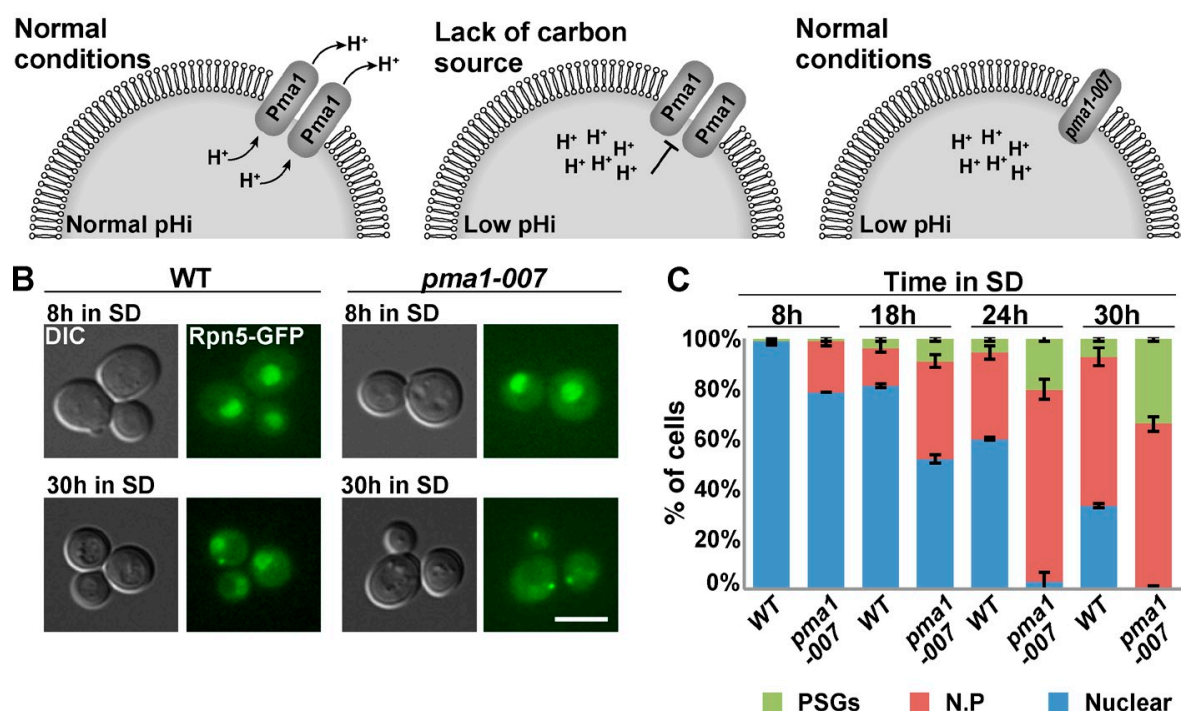

suggests that the reformation of PSGs is also mediated by $\mathrm{V}$-ATPase reassembly and that the V-ATPase complex functions as an intracellular glucose sentinel. Failure of the VMA mutants to sense the changes in glucose levels misregulates cytosolic $\mathrm{pH}$ levels and leads to reduced efficiency of PSGs dynamics.

\section{Drop in pHi affects additional starvation markers}

Previous studies have shown that many diffusely localized cytosolic proteins relocalize into cytoplasmic foci, such as PSGs or actin bodies upon carbon exhaustion (Sagot et al., 2006; Laporte et al., 2008). Because we observed that drop in $\mathrm{pHi}$ as a result of carbon source exhaustion triggers the formation of PSGs, we wanted to test whether pHi mediates the signal for a larger spectrum of cellular reprogramming that takes place upon glucose deprivation. To test this idea, we asked whether pmal-007 mutants also affected changes in actin structure. Indeed, after $8 \mathrm{~h}$ in $\mathrm{SD}$, when the proteasomes are still localized into the nucleus, actin cables and patches were polarized in structures similar to those previously described in proliferating cells in 92\% of the cells (Fig. 5, A and C; Pruyne et al., 2004). In contrast, after $30 \mathrm{~h}$, when PSGs appeared in $40 \%$ of the cells as a result of drop in $\mathrm{pHi}$, the actin cytoskeleton reorganized into structures typical of cells exposed to low carbon source (Sagot et al., 2006), including 65\% showing depolarization of actin patches and $31 \%$ that formed actin bodies (Fig. 5, $\mathrm{B}$ and $\mathrm{C}$ ). The kinetics of actin cytoskeleton reorganization is shown in Fig. 5 C. Similarly, using a stationary-phase granule (SPG) marker, Hos2-GFP (Liu et al., 2012), we found increased formation of SPGs both in pmal-007 and in $\Delta v m a 2$ mutants (Fig. 5, D and E). Similar results were obtained when we analyzed the localization of Hos2-GFP 120 min after cells were shifted to CCCP-containing buffers (similar to the experiment described in Fig. 3 A). The results clearly show that after $120 \mathrm{~min}$, under normal $\mathrm{pH}$ (7.5), the GFP signal was mainly localized to nucleus ( $\sim 75 \%$ of the cells). In contrast, at a lower $\mathrm{pH}$, Hos2-GFP localized to the nuclear periphery or formed SPGs in $76 \%$ of the cells.
These results suggest that, similar to PSGs, the effect on Hos2GFP in pmal-007 and $\Delta v m a 2$ mutants is the result of low cytosolic pH (Fig. S2 D).

Collectively, the relocalization of actin cytoskeleton and Hos2 suggests that cytosolic acidification represents a general signal that affects not only PSG formation but more broadly may be the signal for cytosolic protein relocalization in response to carbon source exhaustion. Cytosolic $\mathrm{pH}$ is regulated not only by glucose but also by other fermentable carbon sources (Dechant et al., 2010). This suggests that the yeast cell may use cytosolic $\mathrm{pH}$ as a downstream reporter on the availability of various carbon sources and enables cells to avoid monitoring the abundance of a specific carbon source or metabolite.

\section{Materials and methods}

Yeast strains and growth conditions

All the strains used in this study are isogenic to BY4741, BY4742, or BY4743 (Brachmann et al., 1998). The relevant genotypes are presented in Table S1. Gene deletions, GFP, and mCherry fusions were performed using one-step PCR-mediated homologous recombination (Longtine et al., 1998; Goldstein and McCusker, 1999). For all deletions, the selection markers replaced the coding region of the targeted genes. GFP and mCherry were fused at the $3^{\prime}$ of the coding region of the targeted genes, by replacing their stop codons. pma 1-007 strain was a gift from C. Lowen's laboratory (University of British Columbia, Vancouver, British Columbia, Canada). In this strain, insertion of the KanMX selection marker upstream of the coding region of $P M A 1$ resulted in reduced activity of the Pmal protein by $50 \%$. We have verified that the proteasome is expressed at the same level

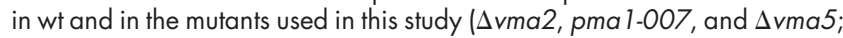
Fig. S1 A). We also show that the expression of the fusion proteins Rpn5GFP, Pup2-GFP, and Pre6-mCherry have no effect on proteasome activity (Fig. S1 B).

Yeast cells were grown in synthetic medium (SD; $0.17 \%$ yeast nitrogen base, $0.5 \%\left(\mathrm{NH}_{4}\right)_{2} \mathrm{SO}_{4}, 2 \%$ glucose, and amino acids). Glucose starvation was performed in SC (SD without glucose). Unless stated otherwise, the $\mathrm{pH}$ of the synthetic media was buffered to $\mathrm{pH}$ 4.6. Yeast cells were grown in liquid medium at $30^{\circ} \mathrm{C}$ in glass tubes. For logarithmic culture, yeast cells were grown for $16-18 \mathrm{~h}$ and then back diluted $10 \times$ with fresh media and allowed to grow for $2 \mathrm{~h}$. For glucose starvation experiments, strains were grown in medium containing $2 \%$ glucose to logarithmic phase, collected by centrifugation, washed once, and resuspended in the same medium lacking glucose. 
A
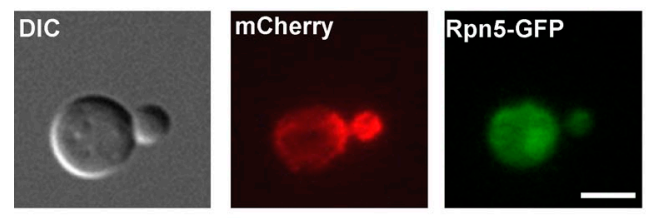

B

t-30h: pma1-007 depolarized cells
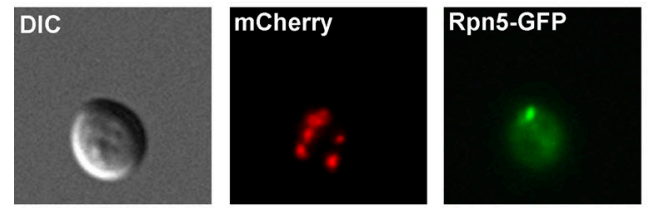

t-30h: pma1-007 actin bodies
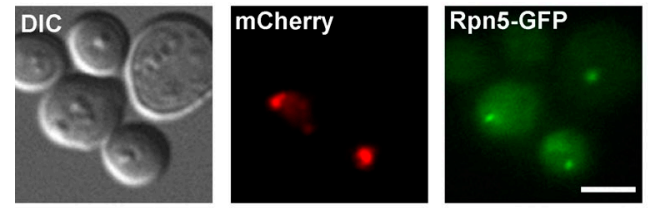

C

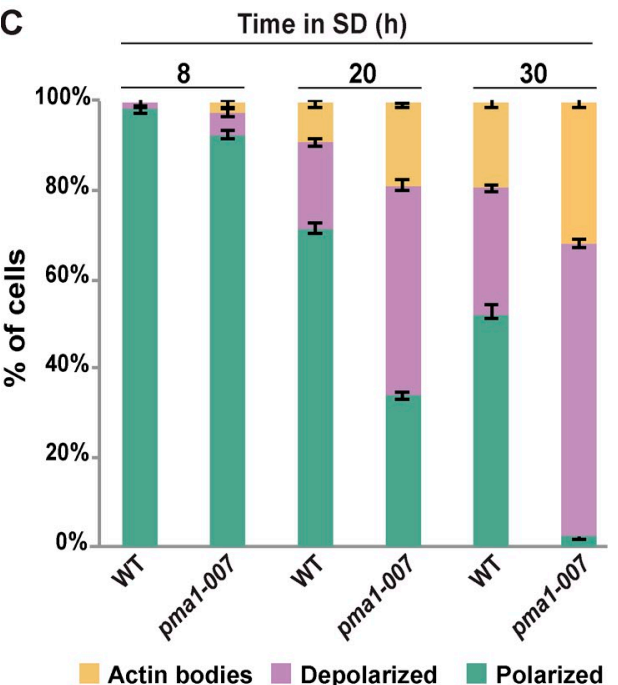

D

WT
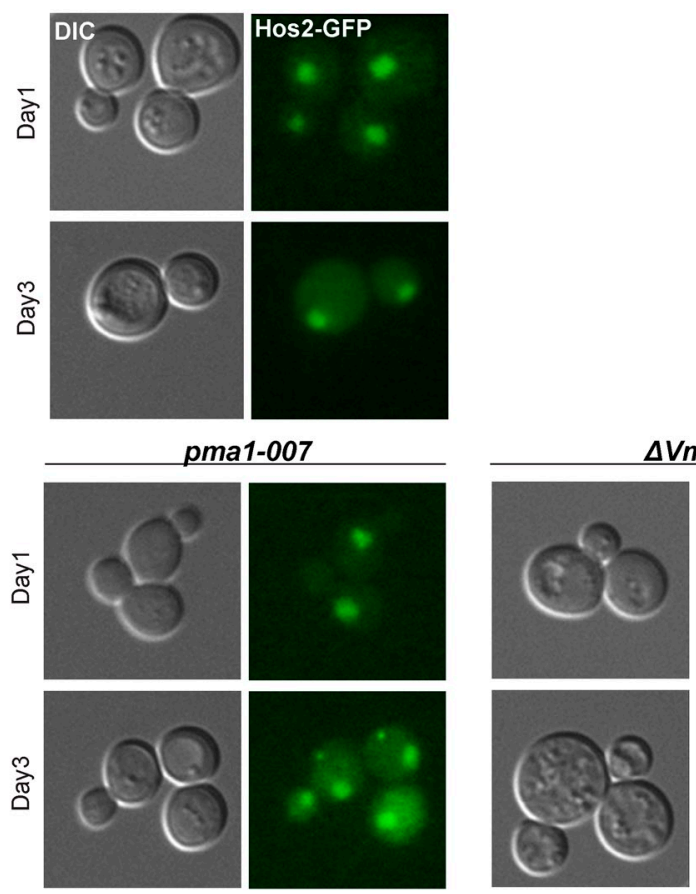

$\Delta V m a 2$
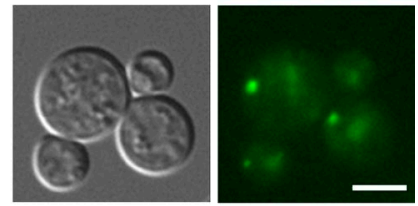

E Time in SD (d)

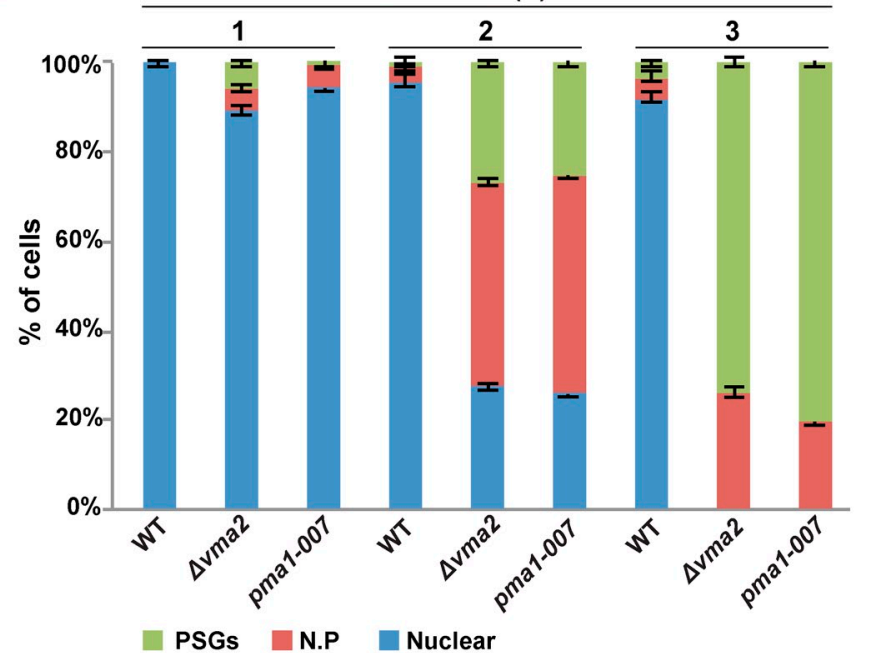

Figure 5. Impaired capacity to control pHi in pma l-007 mutants results in actin cytoskeleton reorganization. pma 1-007 mutants were grown in SD for $30 \mathrm{~h}$. At the indicated time points, samples were taken, stained with Alexa Fluor-phalloidin, and visualized by DIC, GFP (Rpn5-GFP), and mCherry (actin). (A) Examples of polarized actin when most cells $(95 \%)$ show proteasome nuclear localization (time point $8 \mathrm{~h}, n>100)$. (B) Examples of actin reorganization (time point $30 \mathrm{~h}$ ) in cells with PSGs (40\% of the cells, $n>100$ ). (top) Examples for depolarized cells (65\% of the PSG-containing cells). (bottom) Examples for cells with actin bodies (31\% of the PSG-containing cells). (C) Quantitation of the localization patterns for the experiment described in A and B. The localization of actin at the indicated time points was scored as actin bodies, depolarized actin, or polarized actin. Impaired capacity to control pHi results in SPG formation. (D) Logarithmic wt, $\Delta v \mathrm{vma2}$, and pma 1-007 cells expressing the SPG marker Hos2-GFP were washed and resuspended in glucose-containing medium $(t=0)$ for $3 \mathrm{~d}$. Cells were visualized by DIC and GFP. The images represent the dominant pattern at the first and third day. (E) The Hos2-GFP localization at the indicated time points was scored as nuclear, nuclear periphery (N.P), or SPGs. Error bars show the standard deviation between two independent experiments. Bars, $5 \mu \mathrm{m}$.

\section{CCCP treatment}

CCCP treatment was performed as previously described (Orii et al., 2009). In brief, cells were reinoculated at an $\mathrm{OD}_{600 \mathrm{~mm}}$ of 0.6-1 to Hepes buffer (25 mM Hepes, pH 7.4, $200 \mathrm{mM} \mathrm{KCl}, 1 \mathrm{mM} \mathrm{CaCl}$, and $2 \%$ dextrose, $\mathrm{pH} 4.0$ and 7.5) in the presence of $100 \mu \mathrm{M} \mathrm{CCCP}$ (Sigma-Aldrich).

\section{Actin staining}

Actin staining with phalloidin was performed as previously described (Sagot et al., 2006). In brief, cells were fixed with formaldehyde $3.7 \%$ final), washed, and stained overnight with Alexa Fluor 568-phalloidin
(Invitrogen). Cells were then washed twice, resuspended in a mounting solution containing $70 \%$ glycerol and $5 \mathrm{mg} /$ liter paraphenylenediamine, and imaged at room temperature.

\section{Microscopy}

Cells were observed in a fully automated inverted microscope (Axio Observer.Z1; Carl Zeiss) equipped with an automated stage (MS-2000; Applied Scientific Instrumentation), a 300-W xenon light source (Lambda DG-4 LS; Sutter Instrument), a 63x oil, 1.4 NA Plan Apochromat objective lens, and a six-position filter cube turret with a GFP filter (excitation, bp 
$470 / 40 \mathrm{~nm}$; emission, bp 525/50 nm; beamsplitter, FT $495 \mathrm{~nm}$ ) and an HcRed filter (excitation, bp 592/24 nm; emission, bp 675/100 nm; beamsplitter, FT $615 \mathrm{~nm}$ ) obtained from Chroma Technology Corp. Images were acquired using a camera (CoolSnap $\mathrm{HQ}^{2}$; Roper Scientific). The microscope, camera, and shutters (Uniblitz) were controlled by AxioVision Rel. 4.8.2 (Carl Zeiss). Images are a single plane of $z$ stacks performed using a $0.5-\mu \mathrm{m}$ step.

\section{Insertion of Rpn5-GFP to the deletion library}

The SGA technique was performed as described in the following paragraph, to allow efficient introduction of Rpn5-GFP into the yeast deletion collection (for more details see Tong and Boone, 2006). To manipulate the collection in high-density format (384-well plates), we used a bench top colony arrayer (RoToR; Singer Instruments). Specifically, a MATa haploid strain harboring Rpn5-GFP::URA3 integrated into the endogenous locus (Ben-Aroya et al., 2010) and the required markers for SGA (can $1 \Delta:: S T E 2 p r-h i s 5$ and lyp $1 \Delta$ ) were mated on rich media plates against the deletion collection. Diploid cells were selected on plates lacking uracil and provided with G418. Sporulation was then induced by transferring cells to nitrogen starvation plates for $5 \mathrm{~d}$. Haploid cells containing all desired mutations were selected by transferring cells to plates containing all selection markers alongside the toxic amino acid derivatives Canavanine and Thialysine (Sigma-Aldrich) to select against remaining diploids and, lacking histidine, to select for MATa haploid spores. SGA procedure was validated by inspecting representative strains for the presence of the GFP-tagged Rpn5 and deletion of the chosen genes.

\section{High-throughput fluorescence microscopy}

Microscopic screening was performed using an automated microscopy setup as previously described (Herzig et al., 2012). Cells were moved from agar plates into liquid 384-well polystyrene growth plates using the RoToR arrayer. Liquid cultures were grown overnight in SD medium in a shaking incubator (LiCONiC Instruments) at $30^{\circ} \mathrm{C}$. A liquid handler (JANUS; PerkinElmer), which is connected to the incubator, was used to back dilute the strains to $\sim 0.25 \mathrm{OD}$ into plates containing the same medium. Plates were then transferred back to the incubator and were allowed to grow for $3.5 \mathrm{~h}$ at $30^{\circ} \mathrm{C}$ to reach logarithmic growth phase as was validated in preliminary calibration. The liquid handler was then used to transfer strains into glass-bottom 384-well microscope plates (MatriCal Bioscience) coated with Concanavalin A (Sigma-Aldrich) to allow cell adhesion. Wells were washed twice in medium to remove floating cells and reach cell monolayer. Plates were then transferred into an automated inverted fluorescent microscopic scan^R system (Olympus) using a swap robotic arm (Hamilton Robotics). The scan ${ }^{\wedge} R$ system is designed to allow autofocus and imaging of plates in 384-well format using a 60x air lens and is equipped with a cooled charge-coupled device camera (ORCA-ER; Hamamatsu Photonics). Images were acquired at GFP (excitation at $490 / 20 \mathrm{~nm}$ and emission at 535/50 nm).

\section{Online supplemental material}

Fig. S1 shows controls for the experiments described in Fig. 2. Fig. S2 shows controls for the experiments described in Fig. 4 (A-C) and Fig. 5 (D). Table S1 shows the relevant genotype of the strains used in this study. Online supplemental material is available at http://www.jcb.org/cgi/ content/full/jcb.201211146/DC1.

We thank Martin Kupiec and the Ben-Aroya laboratory members for helpful comments on an earlier version of the manuscript.

The work was supported in part by Human Frontier Science Program career development award grant (career development award 00005/2010) and Israeli Cancer Research Fund career development grant (research career development award 201 1-713) to S. Ben-Aroya. M. Schuldiner was funded by a European Research Council grant (starting grant 26039).

Submitted: 27 November 2012

Accepted: 12 April 2013

\section{References}

Ben-Aroya, S., N. Agmon, K. Yuen, T. Kwok, K. McManus, M. Kupiec, and P. Hieter. 2010. Proteasome nuclear activity affects chromosome stability by controlling the turnover of Mms22, a protein important for DNA repair. PLoS Genet. 6:e1000852. http://dx.doi.org/10.1371/journal.pgen .1000852

Brachmann, C.B., A. Davies, G.J. Cost, E. Caputo, J. Li, P. Hieter, and J.D. Boeke. 1998. Designer deletion strains derived from Saccharomyces cerevisiae S288C: a useful set of strains and plasmids for PCR-mediated gene disruption and other applications. Yeast. 14:115-132. http://dx.doi org/10.1002/(SICI)1097-0061(19980130)14:2<115::AID-YEA204>3 $0 . \mathrm{CO} ; 2-2$

Chan, G., D. Hardej, M. Santoro, C. Lau-Cam, and B. Billack. 2007. Evaluation of the antimicrobial activity of ebselen: role of the yeast plasma membrane H+-ATPase. J. Biochem. Mol. Toxicol. 21:252-264. http://dx.doi .org/10.1002/jbt.20189

Cohen, Y., and M. Schuldiner. 2011. Advanced methods for high-throughput microscopy screening of genetically modified yeast libraries. Methods $\mathrm{Mol}$. Biol. 781:127-159. http://dx.doi.org/10.1007/978-1-61779-276-2_8

Daignan-Fornier, B., and I. Sagot. 2011. Proliferation/Quiescence: When to start? Where to stop? What to stock? Cell Div. 6:20. http://dx.doi.org/10 1186/1747-1028-6-20

Dechant, R., M. Binda, S.S. Lee, S. Pelet, J. Winderickx, and M. Peter. 2010. Cytosolic $\mathrm{pH}$ is a second messenger for glucose and regulates the PKA pathway through V-ATPase. EMBO J. 29:2515-2526. http://dx.doi.org/ 10.1038/emboj.2010.138

Giaever, G., A.M. Chu, L. Ni, C. Connelly, L. Riles, S. Véronneau, S. Dow, A. Lucau-Danila, K. Anderson, B. André, et al. 2002. Functional profiling of the Saccharomyces cerevisiae genome. Nature. 418:387-391. http:// dx.doi.org/10.1038/nature00935

Goldstein, A.L., and J.H. McCusker. 1999. Three new dominant drug resistance cassettes for gene disruption in Saccharomyces cerevisiae. Yeast. 15:1541-1553. http://dx.doi.org/10.1002/(SICI)1097-0061(199910)15 14<1541::AID-YEA476>3.0.CO;2-K

Hershko, A., and A. Ciechanover. 1998. The ubiquitin system. Annu. Rev. Biochem. 67:425-479. http://dx.doi.org/10.1146/annurev.biochem.67 .1 .425

Herzig, Y., H.J. Sharpe, Y. Elbaz, S. Munro, and M. Schuldiner. 2012. A systematic approach to pair secretory cargo receptors with their cargo suggests a mechanism for cargo selection by Erv14. PLoS Biol. 10:e1001329. http://dx.doi.org/10.1371/journal.pbio.1001329

Hughes, A.L., and D.E. Gottschling. 2012. An early age increase in vacuolar $\mathrm{pH}$ limits mitochondrial function and lifespan in yeast. Nature. 492:261265. http://dx.doi.org/10.1038/nature11654

Krogan, N.J., M.H. Lam, J. Fillingham, M.C. Keogh, M. Gebbia, J. Li, N. Datta, G. Cagney, S. Buratowski, A. Emili, and J.F. Greenblatt. 2004. Proteasome involvement in the repair of DNA double-strand breaks. Mol. Cell. 16:1027-1034. http://dx.doi.org/10.1016/j.molcel.2004.11.033

Laporte, D., B. Salin, B. Daignan-Fornier, and I. Sagot. 2008. Reversible cytoplasmic localization of the proteasome in quiescent yeast cells. J. Cell Biol. 181:737-745. http://dx.doi.org/10.1083/jcb.200711154

Laporte, D., A. Lebaudy, A. Sahin, B. Pinson, J. Ceschin, B. Daignan-Fornier, and I. Sagot. 2011. Metabolic status rather than cell cycle signals contro quiescence entry and exit. J. Cell Biol. 192:949-957. http://dx.doi.org/ 10.1083/jcb.201009028

Liu, I.C., S.W. Chiu, H.Y. Lee, and J.Y. Leu. 2012. The histone deacetylase Hos2 forms an Hsp42-dependent cytoplasmic granule in quiescent yeast cells.Mol.Biol.Cell.23:1231-1242.http://dx.doi.org/10.1091/mbc.E11-090752

Longtine, M.S., A. McKenzie III, D.J. Demarini, N.G. Shah, A. Wach, A. Brachat, P. Philippsen, and J.R. Pringle. 1998. Additional modules for versatile and economical PCR-based gene deletion and modification in Saccharomyces cerevisiae. Yeast. 14:953-961. http://dx.doi.org/10.1002/ (SICI)1097-0061(199807)14:10<953::AID-YEA293>3.0.CO;2-U

Martínez-Muñoz, G.A., and P. Kane. 2008. Vacuolar and plasma membrane proton pumps collaborate to achieve cytosolic $\mathrm{pH}$ homeostasis in yeast. J. Biol. Chem. 283:20309-20319. http://dx.doi.org/10.1074/jbc .M710470200

Narayanaswamy, R., M. Levy, M. Tsechansky, G.M. Stovall, J.D. O'Connell, J. Mirrielees, A.D. Ellington, and E.M. Marcotte. 2009. Widespread reorganization of metabolic enzymes into reversible assemblies upon nutrient starvation. Proc. Natl. Acad. Sci. USA. 106:10147-10152. http://dx.doi .org/10.1073/pnas.0812771106

Orij, R., J. Postmus, A. Ter Beek, S. Brul, and G.J. Smits. 2009. In vivo measurement of cytosolic and mitochondrial $\mathrm{pH}$ using a $\mathrm{pH}$-sensitive GFP derivative in Saccharomyces cerevisiae reveals a relation between intracellular $\mathrm{pH}$ and growth. Microbiology. 155:268-278. http://dx.doi.org/ 10.1099/mic.0.022038-0

Porat, Z., N. Wender, O. Erez, and C. Kahana. 2005. Mechanism of polyamine tolerance in yeast: novel regulators and insights. Cell. Mol. Life Sci. 62:3106-3116. http://dx.doi.org/10.1007/s00018-005-5341-7

Pruyne, D., A. Legesse-Miller, L. Gao, Y. Dong, and A. Bretscher. 2004. Mechanisms of polarized growth and organelle segregation in yeast. Annu. Rev. Cell Dev. Biol. 20:559-591. http://dx.doi.org/10.1146/annurev cellbio.20.010403.103108

Russell, S.J., K.A. Steger, and S.A. Johnston. 1999. Subcellular localization, stoichiometry, and protein levels of $26 \mathrm{~S}$ proteasome subunits in 
yeast. J. Biol. Chem. 274:21943-21952. http://dx.doi.org/10.1074/jbc .274 .31 .21943

Sagot, I., B. Pinson, B. Salin, and B. Daignan-Fornier. 2006. Actin bodies in yeast quiescent cells: an immediately available actin reserve? Mol. Biol. Cell. 17:4645-4655. http://dx.doi.org/10.1091/mbc.E06-04-0282

Tong, A.H., and C. Boone. 2006. Synthetic genetic array analysis in Saccharomyces cerevisiae. Methods Mol. Biol. 313:171-192.

Young, B.P., J.J. Shin, R. Orij, J.T. Chao, S.C. Li, X.L. Guan, A. Khong, E. Jan, M.R. Wenk, W.A. Prinz, et al. 2010. Phosphatidic acid is a pH biosensor that links membrane biogenesis to metabolism. Science. 329:1085-1088. http://dx.doi.org/10.1126/science.1191026 